\begin{tabular}{|c|c|c|}
\hline (1) & $\begin{array}{c}\text { Türkiye Tarımsal Araştırmalar Dergisi } \\
\text { dergipark.org.tr/tutad }\end{array}$ & $\begin{array}{l}\text { Turk J Agric Res } \\
\text { 2020, 7(1): 59-65 } \\
\text { ๑ TÜTAD } \\
\text { ISSN: 2148-2306 } \\
\text { e-ISSN: 2528-858X }\end{array}$ \\
\hline $\begin{array}{l}\text { UNIVERSiTESI } \\
\text { Bilimin Dyüunda }\end{array}$ & Research Article & $\begin{array}{l}\text { e-ISSN: } 2528-858 \mathrm{X} \\
\text { doi: } 10.19159 / \text { tutad.657668 }\end{array}$ \\
\hline
\end{tabular}

\title{
Genotypic Variation in Calcium Uptake in Common Bean (Phaseolus vulgaris L.) under Chilling Stress
}

\author{
Sultan DERE ${ }^{1 *}$, Hayriye Yıldiz DAŞGAN ${ }^{2}$, Elife CEYLAN² ${ }^{2}$ Merve ÍLHAN ${ }^{2}$ \\ ${ }^{\prime}$ Siirt University, Faculty of Agriculture, Department of Horticulture, Siirt, TURKEY \\ ${ }^{2}$ Çukurova University, Faculty of Agriculture, Department of Horticulture, Adana, TURKEY
}

\begin{tabular}{|c|c|}
\hline & Accepted: 29.02 .2020 \\
\hline \multicolumn{2}{|l|}{ ORCID ID (by author order) } \\
\hline (D) orcid.org/0000-0001-5928-1060 & (D)orcid.org/0000-0003-4578-5553 Dorcid.org/0000-0002-0134-0571 \\
\hline (1D) orcid.org/0000-0001-6506-5970 & \\
\hline
\end{tabular}

\begin{abstract}
Plants are constantly exposed to different abiotic stresses throughout their lifecycle. Sub-optimal temperatures are important abiotic stress factors in agriculture. Plant growth, development, yield, and crop quality are significantly affected by low temperature stress. In the present study, 95 different common bean genotypes were grown in the growth chamber. As the control group, the growth chamber was designed to be $16 / 8 \mathrm{~h} \mathrm{light} \mathrm{and} \mathrm{dark} \mathrm{with} 25^{\circ} \mathrm{C}$ and $20^{\circ} \mathrm{C}$ day/night temperatures, respectively. Fifteen days old seedlings were exposed to chilling stress as cold stress treatment in the dark (night) at $4{ }^{\circ} \mathrm{C}$ for 8 hours and in light (day) at $25^{\circ} \mathrm{C}$ for 16 hours. After fifteen days of low temperature stress, the experiment was completed to investigate calcium $(\mathrm{Ca})$ intake in plants. Shoot $\mathrm{Ca}$ concentration of the genotypes were analyzed and genotypes were classified according to their $\mathrm{Ca}$ content. Therefore 24 genotypes were found to have less than $\leq 0.5 \%, 35$ genotypes were found to have between 0.51 to $1.0 \%$ and 36 genotypes were found to have between 1.01 to $3.0 \%$ Ca content under chilling stress.
\end{abstract}

Keywords: Cold stress, bean, calcium, abiotic stress, low temperature

\section{Introduction}

Climate change has been discusssed and evaluated since the late 1980s. Many aspects of the climate change has been evaluated by taking into account the internal and external forces and factors that may cause changes in the global climate system, as well as human activities that increase greenhouse gas accumulations (Türkeş, 2003).

Scientists have a great deal of consensus that climate is changing and will continue to be affected by future impacts. The adverse effects of these changes in the climate will affect the people who are already disadvantaged due to their region and living conditions. Especially low income farmers relying on agriculture and living in rural areas feel more destructive effects of climate change.

Stress is defined as the damaging power of various factors on plants. Stress causes changes in the growth and development of plants. Stress occurs at any time in the life of the plant and adversely affects the plants, causing the death of plants, organs or tissues.

For each plant to have normal growth and development, a certain optimum temperature range is required and temperatures below this are perceived as cold stress. Besides, certain temperature conditions may be optimal for a plant species and cause stress to the other (Mahajan and Tuteja, 2005).

Approximately $25 \%$ of the terrestrial area on the Earth is composed of regions that do not fall below $15^{\circ} \mathrm{C}$ and are reliable for frost damage. Particularly cold-sensitive plants can be damaged due to the temperature falling below $0{ }^{\circ} \mathrm{C}$ in periods in the remaining areas (Sakai ve Larcher, 1987; Scebba et al., 1998; Zhao, 1998; Vagujfalvi et al., 1999; Pearce, 1999, 2001; Szalai et al., 2001; Puhakainen, 2004).

Signs of cold damage vary depending on the duration of exposure to cold on the plant 
(genotype), the developmental stage, cold contact tissue, wind, water, nutrients and other environmental factors such as light (Saltveit and Morris, 1990).

In general, these symptoms cause reduced growth rate, and leaf width, while increase in cellular autolysis and aging, programmed cell death, chlorosis loss due to photo-oxidation, deterioration of cell membrane structures, consequent disruption of cellular integrity, reduced protoplasmatic flow and necrosis (Kratsch and Wise, 2000; Mahajan and Tuteja, 2005; Rymen et al., 2007).

Under low temperature stress, plants show a variety of cold-induced physiological and biochemical responses, such as the production of reactive oxygen species (ROS), changes in membrane lipid composition and changes in osmolytes (Thomashow, 1999; Browse and Xin, 2001). The mechanism of tolerance to chilling stress in plants is a complex network that is influenced by many factors. There is a need for indepth evaluation of cold stress response mechanisms. Intra and intercellular frost formation mechanisms, frost formation in the tissues and dry matter content change, as well as lipids, proteins, some amino acids, antioxidant enzymes, macro, and micronutrients are thought to be important factors for the examination of changes in elements.

Calcium $(\mathrm{Ca})$ acts as a secondary signal in plants against external impacts (Trewavas and Malhó, 1997). Various biotic and abiotic stimulants such as chilling, touch, light, pathogens, and plant hormones cause a temporary increase in cytosolic Ca concentration (Knight and Knight, 2000; ScraseField and Knight, 2003; Turan, 2007). The earliest response to low temperature was reported to be the temporary increase in free cytosolic $\mathrm{Ca}$ concentration (Monroy and Dhindsa, 1995; Turan, 2007).

Phaseolus sp. can undergo substantial hardening or acclimatization to low temperatures. Plant growth decreases in chilling stress. The activities of superoxide dismutase, ascorbate peroxidase, and glutathione reductase were increased in French bean under chilling stress, while $\beta$-carotene content was decreased (El-Saht, 1998).

The aim of the present study was to determine the effect of chilling stress on $\mathrm{Ca}$ content in common bean genotypes. Thus, bean genotypes were classified according to $\mathrm{Ca}$ content under chilling stress.

\section{Materials and Methods}

The experiment was conducted in a growth chamber of the Department of Horticulture, Faculty of Agriculture at Çukurova University, Adana, Turkey. In the experiment, seedlings of common bean were studied. 95 different bean (Phaseolus vulgaris L.) genotypes were grown in the growth chamber under control and chilling stressed conditions. The seeds of the bean genotypes were sown in 2-liter pots containing vermiculite. For each genotype, 2 pots and 4 plants were grown in each replication. The growth chamber for the control treatments were arranged to $25 / 20^{\circ} \mathrm{C}$ for $16 / 8$ hours in light and dark, respectively. The plants were grown in these conditions for 15 days. After $15^{\text {th }}$-day, plants were exposed to $25 / 4{ }^{\circ} \mathrm{C}$ for $16 / 8$ hours of light and dark, respectively. The nutrient requirements of the plants were supplied by irrigating with the nutrient solution. The mineral content of the nutrient solution is shown in Table 1.

Table 1. Total element concentrations in the nutrient solution used in the experiment

\begin{tabular}{lc}
\hline Elements & Concentration $\left(\mathrm{mg} \mathrm{L}^{-1}\right)$ \\
\hline Nitrogen & 130 \\
Phosphorus & 35 \\
Potassium & 220 \\
Magnesium & 45 \\
Calcium & 150 \\
Sulfur & 70 \\
Iron & 1.5 \\
Manganese & 0.8 \\
Boron & 0.5 \\
Zinc & 0.15 \\
Copper & 0.10 \\
Molybdenum & 0.10 \\
\hline
\end{tabular}

The shoot of the plants was washed once with tap water and twice using deionized water. They were then dried in a forced-air oven at $65^{\circ} \mathrm{C}$ for 48 hours and were ground (40 mesh sieve) for elemental analysis. Ground samples were dry shed in a muffle furnace at $550{ }^{\circ} \mathrm{C}$ for $6 \mathrm{~h}$. The ash was then dissolved in $0.1 \mathrm{M} \mathrm{HCl}$ (hydrochloric acid) solution. Calcium concentrations were determined using an atomic absorption spectrophotometer (Jones, 2001).

The experiment was designed based on completely randomized experimental design. All data presented for $\mathrm{Ca}$ content were the average values of four replications of each treatment. Data were analyzed using JMP 7 software (SAS Institute Inc.). Independent samples t-test were used for comparison of plant $\mathrm{Ca}$ content under control and chilling stressed conditions. The data were also subjected to percent changes under chilling stress relative to control. 


\section{Results and Discussion}

It was shown that chilling stress affected shoot $\mathrm{Ca}$ content of the common bean genotypes evaluated in this study. While Ca content of some genotypes was increased, some of them were stable or decreased.

The $\mathrm{Ca}$ concentration under chilling stress relative to control were higher in $\mathrm{Bn}-70, \mathrm{Bn}-75 \mathrm{~B}$, Bn-153, Bn-103, Bn-72, Bn-76, Bn-6, Bn-62, Bn$114, \mathrm{Bn}-30$, and $\mathrm{Bn}-127$, respectively (Table 2). However, Ca concentration under chilling stress relative to control were lower in $\mathrm{Bn}-106, \mathrm{Bn}-131$, Bn-74, Bn-57, Bn-63, Bn-15, Bn-16, Bn-29, Bn-32, $\mathrm{Bn}-24, \mathrm{Bn}-112$, and $\mathrm{Bn}-27$, respectively.
When control and chilling stressed treatments were compared in terms of shoot $\mathrm{Ca}$ content by $\mathrm{t}$ test, differences of twenty-six genotypes were not statistically significant. Ca concentration increases of chilled bean genotypes relative to control was observed between 2.11 to $478.13 \%$. Ca concentration reduction on chilling stressed bean genotypes relative to control was between 1.69 and 96.1\% (Table 2).

Under chilling stress, the best $\mathrm{Ca}$ contents were found in Bn-114, Bn-13, Bn-37, Bn-163A, and Bn35 , respectively. However, the lowest $\mathrm{Ca}$ contents were found in Bn-27, Bn-99, Bn-151, Bn-2, and Bn15 , respectively (Table 2 ).

Table 2. Shoot Ca concentration of the bean genotypes in control and chilling stress

\begin{tabular}{|c|c|c|c|c|}
\hline Genotypes & $\begin{array}{c}\text { Control } \\
(\%)\end{array}$ & $\begin{array}{c}\text { Chilled } \\
(\%)\end{array}$ & t-test & $\begin{array}{c}\text { Change in Ca concentration } \\
\text { relative to control }\end{array}$ \\
\hline $\mathrm{Bn}-2$ & $0.21 \pm 0.02$ & $0.31 \pm 0.01$ & $0.0072^{*}$ & 47.62 \\
\hline $\mathrm{Bn}-4$ & $0.62 \pm 0.10$ & $0.35 \pm 0.02$ & $0.0507^{\mathrm{ns}}$ & -43.55 \\
\hline Bn-6 & $0.43 \pm 0.03$ & $1.02 \pm 0.02$ & $<.0001^{*}$ & 137.21 \\
\hline $\mathrm{Bn}-8$ & $2.93 \pm 0.41$ & $1.26 \pm 0.00$ & $0.0002^{*}$ & -57.00 \\
\hline $\mathrm{Bn}-9$ & $0.60 \pm 0.22$ & $0.72 \pm 0.01$ & $0.4228^{\mathrm{ns}}$ & 20.00 \\
\hline $\mathrm{Bn}-10$ & $1.48 \pm 0.21$ & $2.01 \pm 0.01$ & $0.0021^{*}$ & 35.81 \\
\hline $\mathrm{Bn}-12$ & $1.72 \pm 0.37$ & $1.60 \pm 0.11$ & $0.7223^{\mathrm{ns}}$ & -6.98 \\
\hline $\mathrm{Bn}-13$ & $1.66 \pm 0.16$ & $2.50 \pm 0.04$ & $0.0190^{*}$ & 50.60 \\
\hline Bn-14 & $1.47 \pm 0.01$ & $1.28 \pm 0.06$ & $0.3318^{\mathrm{ns}}$ & -12.93 \\
\hline $\mathrm{Bn}-15$ & $1.16 \pm 0.01$ & $0.32 \pm 0.02$ & $<.0001^{*}$ & -72.41 \\
\hline Bn-16 & $2.03 \pm 0.00$ & $0.68 \pm 0.07$ & $<.0001^{*}$ & -66.50 \\
\hline $\mathrm{Bn}-17$ & $0.76 \pm 0.04$ & $0.46 \pm 0.05$ & $<.0004^{*}$ & -39.47 \\
\hline Bn-18 & $1.18 \pm 0.00$ & $1.65 \pm 0.12$ & $0.0054^{*}$ & 39.83 \\
\hline Bn-19 & $1.53 \pm 0.07$ & $1.69 \pm 0.02$ & $0.0790^{\mathrm{ns}}$ & 10.46 \\
\hline $\mathrm{Bn}-20$ & $2.16 \pm 0.08$ & $1.57 \pm 0.23$ & $0.0002^{*}$ & -27.31 \\
\hline $\mathrm{Bn}-22$ & $2.24 \pm 0.09$ & $1.68 \pm 0.11$ & $<.0001^{*}$ & -25.00 \\
\hline $\mathrm{Bn}-23$ & $3.43 \pm 0.20$ & $1.54 \pm 0.00$ & $<.0001^{*}$ & -55.10 \\
\hline $\mathrm{Bn}-24$ & $2.27 \pm 0.03$ & $0.83 \pm 0.16$ & $<.0001^{*}$ & -63.44 \\
\hline $\mathrm{Bn}-26$ & $1.67 \pm 0.03$ & $1.45 \pm 0.19$ & $0.2827^{\mathrm{ns}}$ & -13.17 \\
\hline $\mathrm{Bn}-27$ & $0.71 \pm 0.03$ & $0.28 \pm 0.00$ & $<.0001^{*}$ & -60.56 \\
\hline $\mathrm{Bn}-28$ & $0.63 \pm 0.00$ & $0.86 \pm 0.00$ & $0.0157^{*}$ & 36.51 \\
\hline Bn-29 & $1.14 \pm 0.06$ & $0.40 \pm 0.06$ & $<.0001^{*}$ & -64.91 \\
\hline $\mathrm{Bn}-30$ & $0.69 \pm 0.06$ & $1.36 \pm 0.09$ & $0.0012^{*}$ & 97.10 \\
\hline $\mathrm{Bn}-32$ & $1.05 \pm 0.03$ & $0.37 \pm 0.03$ & $<.0001^{*}$ & -64.76 \\
\hline $\mathrm{Bn}-33$ & $1.03 \pm 0.01$ & $0.69 \pm 0.03$ & $0.0080^{*}$ & -33.01 \\
\hline $\mathrm{Bn}-34$ & $0.89 \pm 0.11$ & $0.52 \pm 0.07$ & $0.0051^{*}$ & -41.57 \\
\hline $\mathrm{Bn}-35$ & $2.17 \pm 0.29$ & $2.10 \pm 0.07$ & $0.8051^{\mathrm{ns}}$ & -3.23 \\
\hline $\mathrm{Bn}-36$ & $0.93 \pm 0.19$ & $0.69 \pm 0.09$ & $0.2870^{\mathrm{ns}}$ & -25.81 \\
\hline $\mathrm{Bn}-37$ & $1.33 \pm 0.03$ & $2.25 \pm 0.17$ & $0.0418^{*}$ & 69.17 \\
\hline $\mathrm{Bn}-38$ & $1.53 \pm 0.22$ & $1.78 \pm 0.00$ & $0.3355^{\mathrm{ns}}$ & 16.34 \\
\hline Bn-39 & $1.80 \pm 0.20$ & $0.66 \pm 0.13$ & $0.0004^{*}$ & -63.33 \\
\hline $\mathrm{Bn}-40$ & $0.91 \pm 0.00$ & $1.09 \pm 0.08$ & $0.1402^{\mathrm{ns}}$ & 19.78 \\
\hline $\mathrm{Bn}-42$ & $1.23 \pm 0.00$ & $1.67 \pm 0.13$ & $0.0005^{*}$ & 35.77 \\
\hline $\mathrm{Bn}-44$ & $1.03 \pm 0.07$ & $1.73 \pm 0.01$ & $<.0001^{*}$ & 67.96 \\
\hline $\mathrm{Bn}-48$ & $2.78 \pm 0.08$ & $1.13 \pm 0.08$ & $<.0001^{*}$ & -59.35 \\
\hline$B n-49$ & $1.59 \pm 0.12$ & $1.39 \pm 0.10$ & $0.1200^{\mathrm{ns}}$ & -12.58 \\
\hline $\mathrm{Bn}-50$ & $1.22 \pm 0.03$ & $0.81 \pm 0.11$ & $<.0001^{*}$ & -33.61 \\
\hline $\mathrm{Bn}-51$ & $1.27 \pm 0.03$ & $1.07 \pm 0.07$ & $0.2291^{\mathrm{ns}}$ & -15.75 \\
\hline $\mathrm{Bn}-52 \mathrm{~A}$ & $1.18 \pm 0.14$ & $1.16 \pm 0.00$ & $0.8320^{\mathrm{ns}}$ & -1.69 \\
\hline $\mathrm{Bn}-52 \mathrm{~B}$ & $1.50 \pm 0.07$ & $1.39 \pm 0.13$ & $0.2744^{\mathrm{ns}}$ & -11.46 \\
\hline $\mathrm{Bn}-54$ & $0.43 \pm 0.02$ & $0.68 \pm 0.04$ & $0.0013^{*}$ & 58.14 \\
\hline $\mathrm{Bn}-55$ & $1.24 \pm 0.02$ & $1.34 \pm 0.05$ & $0.3833^{\mathrm{ns}}$ & 8.06 \\
\hline
\end{tabular}

*: Significant, ns: non significant 
Table 2. (continued)

\begin{tabular}{|c|c|c|c|c|}
\hline Genotypes & $\begin{array}{l}\text { Control } \\
(\%)\end{array}$ & $\begin{array}{c}\text { Chilled } \\
(\%)\end{array}$ & t-test & $\begin{array}{l}\text { Change in Ca concentration } \\
\text { relative to control }\end{array}$ \\
\hline $\mathrm{Bn}-56$ & $0.96 \pm 0.03$ & $0.43 \pm 0.18$ & $<.0001^{*}$ & -55.21 \\
\hline Bn-57 & $1.44 \pm 0.06$ & $0.33 \pm 0.19$ & $<.0001^{*}$ & -77.08 \\
\hline Bn-60 & $1.43 \pm 0.14$ & $1.73 \pm 0.23$ & $0.0281^{*}$ & 20.98 \\
\hline Bn-61 & $1.08 \pm 0.11$ & $1.26 \pm 0.00$ & $0.0062^{*}$ & 16.67 \\
\hline Bn-62 & $0.74 \pm 0.01$ & $1.58 \pm 0.13$ & $0.0001^{*}$ & 113.51 \\
\hline Bn-63 & $0.64 \pm 0.06$ & $0.16 \pm 0.02$ & $0.0002^{*}$ & -75.00 \\
\hline Bn-64 & $0.64 \pm 0.13$ & $1.07 \pm 0.18$ & $0.0256^{*}$ & 67.19 \\
\hline Bn-65 & $0.66 \pm 0.05$ & $0.41 \pm 0.03$ & $0.0032^{*}$ & -37.88 \\
\hline Bn-66 & $1.24 \pm 0.08$ & $1.48 \pm 0.14$ & $0.0119^{*}$ & 19.35 \\
\hline Bn-67 & $0.98 \pm 0.19$ & $1.46 \pm 0.11$ & $0.0089^{*}$ & 48.98 \\
\hline Bn-68 & $1.30 \pm 0.02$ & $1.09 \pm 0.08$ & $0.0534^{\mathrm{ns}}$ & -16.15 \\
\hline Bn-69 & $1.31 \pm 0.01$ & $1.00 \pm 0.18$ & $0.0466^{*}$ & -23.66 \\
\hline Bn-70 & $0.32 \pm 0.14$ & $1.85 \pm 0.34$ & $<.0001^{*}$ & 478.13 \\
\hline Bn-71 & $1.09 \pm 0.12$ & $1.20 \pm 0.04$ & $0.5744^{\mathrm{ns}}$ & 10.09 \\
\hline Bn-72 & $0.37 \pm 0.00$ & $1.04 \pm 0.16$ & $<.0001^{*}$ & 181.08 \\
\hline Bn-74 & $0.42 \pm 0.06$ & $0.07 \pm 0.00$ & $<.0001^{*}$ & -83.33 \\
\hline $\mathrm{Bn}-75 \mathrm{~A}$ & $1.02 \pm 0.05$ & $0.52 \pm 0.19$ & $0.0080^{*}$ & -49.02 \\
\hline $\mathrm{Bn}-75 \mathrm{~B}$ & $0.32 \pm 0.07$ & $1.70 \pm 0.02$ & $<.0001^{*}$ & 431.25 \\
\hline $\mathrm{Bn}-75 \mathrm{C}$ & $1.31 \pm 0.01$ & $0.66 \pm 0.10$ & $0.0008^{*}$ & -49.62 \\
\hline Bn-76 & $0.46 \pm 0.06$ & $1.19 \pm 0.08$ & $0.0034^{*}$ & 158.70 \\
\hline Bn-77 & $1.73 \pm 0.08$ & $0.88 \pm 0.11$ & $<.0001^{*}$ & -49.13 \\
\hline Bn-79 & $0.45 \pm 0.09$ & $0.33 \pm 0.04$ & $0.0213^{*}$ & -26.67 \\
\hline Bn-87 & $1.63 \pm 0.05$ & $0.72 \pm 0.12$ & $<.0001^{*}$ & -55.83 \\
\hline Bn-89 & $0.59 \pm 0.04$ & $0.37 \pm 0.05$ & $0.0172^{*}$ & -37.29 \\
\hline Bn-99 & $0.39 \pm 0.02$ & $0.29 \pm 0.08$ & $0.0255^{*}$ & -25.64 \\
\hline Bn-101 & $2.81 \pm 0.01$ & $1.93 \pm 0.15$ & $<.0001^{*}$ & -31.32 \\
\hline Bn-102 & $1.96 \pm 0.16$ & $1.30 \pm 0.07$ & $<.0001^{*}$ & -33.67 \\
\hline Bn-103 & $0.10 \pm 0.00$ & $0.40 \pm 0.16$ & $<.0001^{*}$ & 300.00 \\
\hline Bn-104 & $1.26 \pm 0.18$ & $1.19 \pm 0.02$ & $0.3146^{\mathrm{ns}}$ & -5.56 \\
\hline Bn-105 & $0.65 \pm 0.07$ & $0.43 \pm 0.06$ & $0.0214^{*}$ & -33.85 \\
\hline Bn-106 & $1.54 \pm 0.07$ & $0.06 \pm 0.02$ & $<.0001^{*}$ & -96.10 \\
\hline Bn-111 & $1.18 \pm 0.04$ & $1.22 \pm 0.08$ & $0.6792^{\text {ns }}$ & 3.39 \\
\hline Bn-112 & $0.40 \pm 0.01$ & $0.15 \pm 0.01$ & $<.0001^{*}$ & -62.50 \\
\hline Bn-114 & $1.81 \pm 0.25$ & $3.76 \pm 0.33$ & $<.0001^{*}$ & 107.73 \\
\hline Bn-116 & $2.68 \pm 0.00$ & $1.64 \pm 0.05$ & $<.0001^{*}$ & -38.81 \\
\hline Bn-127 & $0.81 \pm 0.02$ & $1.46 \pm 0.08$ & $<.0001^{*}$ & 80.25 \\
\hline Bn-130 & $1.31 \pm 0.03$ & $1.43 \pm 0.03$ & $0.4573^{\text {ns }}$ & 9.16 \\
\hline Bn-131 & $0.49 \pm 0.00$ & $0.07 \pm 0.16$ & $<.0001^{*}$ & -85.71 \\
\hline Bn-138 & $1.42 \pm 0.03$ & $1.45 \pm 0.07$ & $0.6100^{\mathrm{ns}}$ & 2.11 \\
\hline Bn-139 & $3.21 \pm 0.00$ & $1.41 \pm 0.08$ & $<.0001^{*}$ & -56.07 \\
\hline Bn-140 & $1.52 \pm 0.14$ & $1.84 \pm 0.27$ & $0.0822^{\text {ns }}$ & 21.05 \\
\hline Bn-147 & $0.82 \pm 0.08$ & $0.62 \pm 0.05$ & $<.0001^{*}$ & -24.39 \\
\hline Bn-151 & $0.72 \pm 0.00$ & $0.30 \pm 0.09$ & $<.0001^{*}$ & -58.33 \\
\hline Bn-152 & $0.31 \pm 0.05$ & $0.33 \pm 0.12$ & $0.4732^{\text {ns }}$ & 6.45 \\
\hline Bn-153 & $0.14 \pm 0.04$ & $0.68 \pm 0.09$ & $<.0001^{*}$ & 385.71 \\
\hline Bn-154 & $0.64 \pm 0.02$ & $0.46 \pm 0.27$ & $0.0004^{*}$ & -28.13 \\
\hline $\mathrm{Bn}-157 \mathrm{~A}$ & $1.01 \pm 0.00$ & $0.63 \pm 0.02$ & $<.0001^{*}$ & -37.62 \\
\hline Bn-157B & $1.41 \pm 0.08$ & $1.00 \pm 0.13$ & $0.0678^{\text {ns }}$ & -29.08 \\
\hline Bn-158 & $0.94 \pm 0.08$ & $0.99 \pm 0.21$ & $0.0278^{*}$ & 5.32 \\
\hline Bn-159 & $0.85 \pm 0.13$ & $0.53 \pm 0.17$ & $<.0001^{*}$ & -37.65 \\
\hline Bn-162 & $1.69 \pm 0.16$ & $1.06 \pm 0.02$ & $0.0053^{*}$ & -37.28 \\
\hline $\mathrm{Bn}-163 \mathrm{~A}$ & $1.71 \pm 0.01$ & $2.13 \pm 0.00$ & $0.5890^{\mathrm{ns}}$ & 24.56 \\
\hline Bn-163B & $1.02 \pm 0.07$ & $0.53 \pm 0.11$ & $0.1208^{\text {ns }}$ & -48.04 \\
\hline
\end{tabular}

*: Significant, ns: non significant

The Ca concentration of plants under control treatment ranged between $0.10 \%$ to $3.43 \%$, while it was between $0.06 \%$ to $3.76 \%$ in cold treated plants.

Cold acclimation is important and a universal phenomenon in chilling tolerant plants (Srivastava et al., 2015). Physiological and biochemical changes occur in plants during cold stress (Miura and Furumoto, 2013). The adequate $\mathrm{Ca}$ concentrations in bean leaves are between $0.50 \%$ and $2.00 \%$ under normal conditions (Favaro et al., 
2007). When plants are exposed to low temperature stress, $\mathrm{Ca}$ has been reported to be accumulated temporarily in the tissues of some alfalfa genotypes (Medicago sativa) (Aslantas et al., 2010; Puhakainen, 2004). In this study, chilling stress caused an increase in the Ca concentration on some genotypes. The least shoot $\mathrm{Ca}$ concentration was $0.06 \%$ (Bn106) and the highest was 3.76\% (Bn114) under chilling stress in this study. Under chilling stress, it was reported that there was $\mathrm{Ca}$ flow from the apoplast to the cytoplasm fluid. The Ca channels in plasma membranes acts as sensors in a temperature drop. Ca increase in cells is one important phenomenon for the study of genes that react to low temperature stress (Aslantas et al., 2010; Smallwood and Bowles, 2002). Ca signaling plays a crucial role in conferring chilling tolerance in plants. Generally, plant genotypes that tolerate low temperature stress can maintain high leaf water potential by closing their stomata and preventing transpirational water loss (Wilkinson et al., 2001). Calcium is an essential requirement for chilling induced stomatal closure in chilling tolerant genotypes. It is also believed that ABA-induced stomatal closure is partially mediated by $\mathrm{Ca}$ released from internal guard cell stores or the apoplast (Wilkinson et al., 2001), and this function seems to make $\mathrm{Ca}$ a major contributing factor to chilling tolerance and protection of leaves from dehydration. It has been suggested that $\mathrm{Ca}$ is necessary for recovery from low temperature stress by activating the plasma membrane enzyme ATPase which is required to pump back the nutrients that were lost in cell damage (Palta, 2000).

It has been reported that $\mathrm{Ca}$ flow occurs from the apoplast to the cytoplasm fluid. Calcium channels in plasma membranes have been reported to act as sensors against temperature drop (Smallwood and Bowles, 2002). Additionally, frost resistance is higher in flower buds with high dry matter and $\mathrm{Ca}$ content (Aslantaş, 1999).

Plants have been reported to increase cytoplasmic $\mathrm{Ca}$ as an early response to low temperature stress (Leipner, 2009). Researchers have started to investigate the ways in which free cytosolic $\mathrm{Ca}^{2+}\left[\left(\mathrm{Ca}^{2+}\right)\right.$ cyt $]$ increase is triggered by low temperature stress (Minorsky, 1985) and oxidative stress occurs in low temperature-sensitive plants (Hariyadi and Parkin, 1993). In maize root cells, $\mathrm{Ca}^{+2}$ was reported to increase $20-25 \%$ at a temperature of $2{ }^{\circ} \mathrm{C}$ (Zocchi and Hanson, 1982). The cytosolic free $\mathrm{Ca}$ is a common secondary messenger in abiotic stress signaling. Threats to agriculture such as salinity, water scarcity, and hypoxia are signaled through calcium (Wilkins et al., 2016). Calcium influence on chilling resistance of grafted eggplant seedlings were studied in a hydroponic system. Under the $\mathrm{Ca}$ sufficient treatment, the activities of peroxidase (POD), superoxide dismutase (SOD), and catalase (CAT) in seedlings at $5{ }^{\circ} \mathrm{C}$ were all significantly higher than those of $\mathrm{Ca}$ deficiency. Calcium content was potentially the main reason that grafted seedlings had higher chilling resistance than own-rooted seedlings. Calcium appears to play an important role in increasing the chilling resistance of grafting eggplant seedlings (Gao et al., 2005).

Bean genotypes were classified according to $\mathrm{Ca}$ concent responses under the chilling stress. According to this classification, twenty four bean genotypes were found to have less than $0.50 \%$ or equal, thirty-five bean genotypes were found to have between 0.51 to $1.00 \%$ and thirty-six bean genotypes were found to have between 1.01 to $3.00 \%$ Ca content under chilling stress (Table 3).

Table 3. Classification of bean genotypes according to Ca content under chilling stress

\begin{tabular}{|c|c|c|}
\hline $\begin{array}{c}0.50 \% \leq \mathrm{Ca} \\
\text { content }\end{array}$ & $\begin{array}{c}0.51-1.00 \% \mathrm{Ca} \\
\text { content }\end{array}$ & $\begin{array}{c}1.01-3.00 \% \mathrm{Ca} \\
\text { content }\end{array}$ \\
\hline Bn-2 & Bn-6 & Bn-10 \\
\hline $\mathrm{Bn}-4$ & $\mathrm{Bn}-8$ & Bn-12 \\
\hline Bn-15 & Bn-9 & Bn-13 \\
\hline $\mathrm{Bn}-17$ & $\mathrm{Bn}-16$ & Bn-14 \\
\hline $\mathrm{Bn}-27$ & Bn-24 & Bn-18 \\
\hline Bn-29 & $\mathrm{Bn}-28$ & Bn-19 \\
\hline Bn-32 & Bn-30 & Bn-20 \\
\hline $\mathrm{Bn}-56$ & $\mathrm{Bn}-33$ & Bn-22 \\
\hline Bn-57 & Bn-34 & $\mathrm{Bn}-23$ \\
\hline Bn-63 & $\mathrm{Bn}-36$ & $\mathrm{Bn}-26$ \\
\hline Bn-65 & Bn-39 & $\mathrm{Bn}-35$ \\
\hline Bn-74 & $\mathrm{Bn}-40$ & Bn-37 \\
\hline $\mathrm{Bn}-75 \mathrm{~A}$ & $\mathrm{Bn}-50$ & $\mathrm{Bn}-38$ \\
\hline Bn-79 & $\mathrm{Bn}-51$ & $B n-42$ \\
\hline Bn-89 & $\mathrm{Bn}-54$ & $\mathrm{Bn}-44$ \\
\hline Bn-99 & $\mathrm{Bn}-60$ & $\mathrm{Bn}-48$ \\
\hline Bn-103 & Bn-61 & Bn-49 \\
\hline Bn-105 & $B n-62$ & $\mathrm{Bn}-52 \mathrm{~A}$ \\
\hline Bn-106 & Bn-64 & $\mathrm{Bn}-52 \mathrm{~B}$ \\
\hline Bn-112 & Bn-68 & Bn-55 \\
\hline Bn-131 & Bn-69 & Bn-66 \\
\hline Bn-151 & Bn-72 & Bn-67 \\
\hline Bn-152 & $\mathrm{Bn}-75 \mathrm{C}$ & $\mathrm{Bn}-70$ \\
\hline \multirow[t]{13}{*}{ Bn-154 } & Bn-77 & Bn-71 \\
\hline & Bn-87 & Bn-75 \\
\hline & Bn-104 & $\mathrm{Bn}-76$ \\
\hline & Bn-111 & Bn-101 \\
\hline & Bn-147 & Bn-102 \\
\hline & Bn-153 & Bn-114 \\
\hline & $\mathrm{Bn}-157 \mathrm{~A}$ & Bn-116 \\
\hline & $\mathrm{Bn}-157 \mathrm{~B}$ & Bn-127 \\
\hline & $\mathrm{Bn}-158$ & Bn-130 \\
\hline & Bn-159 & Bn-138 \\
\hline & Bn-162 & Bn-139 \\
\hline & $\mathrm{Bn}-163 \mathrm{~B}$ & Bn-140 \\
\hline & & $\mathrm{Bn}-163 \mathrm{~A}$ \\
\hline
\end{tabular}




\section{Conclusions}

Chilling stress has been shown to affect Ca content in common bean genotypes. Despite the chilling stress, it can be suggested that the genotypes, which have the best $\mathrm{Ca}$ content (Bn-114, Bn-13, Bn-37, $\mathrm{Bn}-163 \mathrm{~A}, \mathrm{Bn}-35)$ can be used in breeding for chilling tolerant cultivars. There is a need for indepth understanding of chilling stress and $\mathrm{Ca}$ interactions due to limited literature about the calcium content of beans. For this reason, our work highlights the chilling and $\mathrm{Ca}$ content relations for future studies.

\section{References}

Aslantaş, R., 1999. Determination of cold hardness degree of flower buds with generative and vegetative groving of some almond (Amygdalus communis L.) cultivars/clones and types in Erzincan conditions. Ph.D. Thesis, Atatürk University Institute of Science, Erzurum, Turkey. (in Turkish).

Aslantas, R., Karakurt, H., Karakurt, Y., 2010. The cellular and molecular mechanisms on resistance to low temperatures in plants. Journal of Agricultural Faculty of Atatürk University, 41(2): 157-167.

Browse, J., Xin, Z., 2001. Temperature sensing and cold acclimation. Current Opinion in Plant Biology, 4: 241-246.

El-Saht, H.M., 1998. Responses to chilling stress in French bean seedlings: antioxidant compounds. Biologia Plantarum, 41: 395-402.

Favaro, S.P., Neto, J.A.B., Takahashi, H.W., Miglioranza, É., Ida, E.I., 2007. Rates of calcium, yreld and quality of snap bean. Scientia Agricola, 64(6): 616-620

Gao, H., Chen, G., Han, L., Lin, H., 2005. Calcium influence on chilling resistance of grafting eggplant Seedlings. Journal of Plant Nutrition, 27(8): 13271339.

Hariyadi, P., Parkin, K.L., 1993. Chilling-induced oxidative stress in cucumber (Cucumis sativus L. cv. Calypso) seedlings. Journal of Plant Physiology, 141(6): 733-738.

Jones, J.B., 2001. Laboratory Guide for Conductivity Soil Tests and Plant Analysis. CRC Press, Taylor and Francis Group, Florida.

Knight, H., Knight, M.R., 2000. Imaging spatial and cellular characteristic of low temperature calcium signature after cold acclimation in Arabidopsis. Journal of Experimental Botany, 51: 1679-1686.

Kratsch, H.A., Wise, R.R., 2000. The ultrastructure of chilling stress. Plant Cell Environment, 23: 337-350.

Leipner, J., 2009. Chilling stress in maize: From physiology to genetics and molecular mechanisms. Habilitation thesis, Department of Agricultural and Food Sciences, Zurich.

Mahajan, S., Tuteja, N., 2005. Cold, salinity and drought stresses. Archives of Biochemistry and Biophysics, 444(2): 139-158.
Minorsky, PV., 1985. A heuristic hypothesis of chilling injury in plants: a role for calcium as the primary physiological transducer of injury. Plant, Cell and Environment, 8: 75-94.

Miura, K., Furumoto, T., 2013. Cold Signaling and cold response in plants. International Journal of Molecular Sciences, 14: 5312-5337.

Monroy, A.F., Dhindsa, R.S., 1995. Low temperature signal transduction: induction of cold acclimationspecific genes of alfalfa by calcium at $25^{\circ} \mathrm{C}$. Plant Cell, 7: 321-331.

Palta, J. P., 2000. Stress interactions at the cellular and membrane levels. Horticulture Science, 25(11): 1377- 1381

Pearce, R.S., 1999. Molecular analysis of acclimation to cold. Plant Growth Regulation, 29: 47-76.

Pearce, R.S., 2001. Plant freezing and damage. Annals of Botany, 87: 417-424.

Puhakainen, T., 2004. Physiological and Molecular Analyses of Cold Acclimation of Plants. Academic dissertation, Genetics Faculty of Biosciences University of Helsinki, Finland.

Reymen, B., Fiorani, F., 2007. Cold night impair leaf growth and cell cycle progression in maize trough transcriptional changes of cell cycle genes. Plant physiology, 143(3): 1429-1438.

Sakai, A., Larcher, W., 1987. Frost Survival of Plants: Response Sand Adaptations to Freezing Stress. Springer-Verlag, New York.

Saltveit, M.E., Morris, L.L., 1990. Overview of chilling injury of horticultural crops. CRC Press, Florida.

Scebba, F., Sebastiani, L., Vitagliano, C., 1998. Changes in activity of antioxidative enzymes in wheat (Triticum aestivum) seedlings under cold acclimation. Phsiologia Plantarum, 104: 747-752.

Scebba, F., Sebastiani, L., Vitagliano, C., 1999. Protective enzymes against activated oxygen species in wheat (Triticum aestivum L.) seedling: Responses to cold acclimation. Journal of Plant Physiology, 155(6): 762-768.

Scrase-Field, S., Knight, M.R., 2003. Calcium; just a chemical switch? Current Opinion in Plant Biology, 6: $1-7$.

Smallwood, M., Bowles, D.J., 2002. Plants in a cold climate. Philosophical Transactions of The Royal Society, 357: 831-847.

Srivastava, V., Soni, A., Sonam, K., 2015. Analysis on effect of cold stress in bean seeds (Phaseolus vulgaris 1). American Journal of BioScience, 3(4): 145-166

Szalai, G., Jvea, T., Paldi, E., Dubacq, J.P., 2001. Changes in the fatty acid unsaturation after hardening in wheat chrosome substitution lines with different cold tolerance. Journal of Plant Physiology, 158: 663-666.

Thomashow, M.F., 1999. Plant cold acclimation: Freezing tolerance genes and regulatory mechanisms. Annual Review Of Plant Physiology And Plant Molecular Biology, 50: 571-599. 
Trewavas, A.J., Malhó, R., 1997. Signal perception and transduction: The origin of the phenotype. Plant Cell, 9: 1181-1195.

Turan, Ö., 2007. Determination of the tolerance of chickpea (Cicer arietinum L.) cultivars and lines to cold stress by physiological and biochemical parameters. Master thesis, Hacettepe University Institute of Science, Ankara, Turkey.

Türkeş, M., 2003. Küresel iklim değişikliği ve gelecekteki iklimimiz. 23 Mart Dünya Meteoroloji Günü Kutlaması Gelecekteki Iklimimiz Paneli, Bildiriler Kitabı, T.C. Çevre ve Orman Bakanlığı Devlet Meteoroloji İșleri Genel Müdürlüğü, 23 Mart, Ankara, s. 12-37.

Vagujfalvi, A., Kerepesi, I., Galiba, G., Tischner, T., Sutka, J., 1999. Frost hardiness depending on carbohydrate changes during cold acclimation in wheat. Plant Science, 144: 85-92.
Wilkins, K.A., Matthus, E., Swarbreck, S. M., Davies, J.D., 2016. Calcium-Mediated Abiotic Stress Signaling in Roots. Frontiers in Plant Science, 7: 1296.

Wilkinson, S., Clephan, A.L., Davies, W.J., 2001. Rapid low temperature-induced stomatal closure occurs in cold-tolerant Commelina communis leaves but not in cold-sensitive tobacco leaves, via a mechanism that involves apoplastic calcium but not abscisic acid. Plant Physiology, 126: 1566-1578.

Zhao, S., 1998. Induction of freezing tolerance in Jack pine seedlings: changes in lipids, oxidation-reduction and antioxidant enzymes during cold acclimation. Ph.D. Thesis, Department of Botany, Toronto.

Zocchi, G., Hanson, J.B., 1982. Calcium influx into corn roots as a results of cold shock. Plant Physiology, 70(318): 319-2968. 